Thorax (1976), 31, 40.

\title{
Cartilage in children's lungs: a quantitative assessment using the right middle lobe
}

\author{
C.C.SINCLA I R-SMITH, JOH N L. EMER Y, \\ D. G A D D O N, F. D INSDALE, a n d J B A D D E L E Y \\ Department of Pathology, Children's Hospital, Sheffield
}

\begin{abstract}
Sinclair-Smith, C. C., Emery, J. L., Gadsdon, D., Dinsdale, F., and Baddeley, J. (1976). Thorax, 31, 40-43. Cartilage in children's lungs: a quantitative assessment using the right middle lobe. The amount and development of cartilage in the lungs of 150 children dying between 24 weeks' gestation and 12 yearrs has been studied using standard sections of the right middle lobe. Both the cartilage-bearing bronchi and the cartilage fragments increase in number until approximately two months after birth, after which time a constant level is maintained. There is a progressive increase in the total area of cartilage in the child's lung during infancy and childhood, and this appears to be more directly related to the crown-rump length of the child than to its age. Percentiles of the quantity of cartilage found in the standard lung sections are presented.
\end{abstract}

The lungs of children dying from a variety of conditions show variable cellularity of the cartilage fragments and present an appearance which suggests that certain factors are influencing the rate of growth of the cartilage. While we have knowledge of gross abnormalities in tracheal and bronchial cartilages recently summarized by Landing and Wells (1973), minor malformations have yet to be defined. To assess the possible importance of abnormal cartilage growth it is necessary to have a clear concept of the normal development of cartilage in children's lungs and a method of routine assessment.

The general structure of the cartilage was described by von Hayek (1960), and the pattern of development of the intrasegmental bronchial cartilage has been studied by Bucher and Reid (1961) and well reviewed by Bucher (1969). Fragments of cartilage become identifiable in the trachea after about 10 weeks of intrauterine life and first appear in the segmental bronchi around the twelfth week. By the sixteenth week all generations of the bronchial tree seen at birth are present. Bucher and Reid (1961) found that cartilage continued to appear until the 25th intrauterine week, and after this there was no further extension of cartilage down the bronchi. They also pointed out that there was a wide variability in the amount of cartilage in different divisions of the respiratory tract although the total number of bronchial generations may be relatively con- stant. Their concern was largely a qualitative on and consisted of serial sections of dissected frage ments of a small number of lungs and exploration of the extent of bronchial cartilages along indis vidual bronchi. Such a method does not lenф itself to routine pathological study where aspects of change in a large number of cases have to be noted.

It has been our practice for many years t⿱ take a standardized block across the whole of the right middle lobe exactly halfway from the hilun to the periphery (Emery and Wilcock, 1966 Emery and Dinsdale, 1972). We report here $\vec{a}$ quantitative assessment of the cartilage in these standard blocks of tissue.

\section{MATERIAL AND METHODS}

The material consisted of a sequential series of 100 right middle lobes from necropsies carried out at the Sheffield Children's Hospital. The ages of the children ranged from 24 weeks' gestatio to 12 years. No exclusion was made of available sections. The right middle lobe of the lung wa $\$$ not dissected for anatomical study if there was an gross congenital deformity of the heart or ob. vious general pulmonary pathology such as maß result from diaphragmatic hernia. When the right middle lobe is completely fused with the right upper lobe as occurs in about 1 in 40 lungs the blocking system is not applicable. Thus the 
gross anatomy of all the lobes was normal. As a sequential series of children coming to necropsy includes only a small proportion of children over the age of 1 year, a sequential series of 50 lungs from children dying over the age of 18 months was also studied.

Sections were stained with a variety of stains including scarba red and alcian blue, haematoxylin and eosin, and Masson's trichrome, but for the majority of assessments scarba red and alcian blue were used.

We first attempted to study the cartilage fragments using a Quantimet 720 Image Analysing Microscope. The whole field of the section was scanned and the area of each fragment of cartilage was measured using the light pen. The second method used a standard projection microscope, the images of the cartilage being outlined on a sheet of high quality paper, then cut out and weighed. The area of each piece was determined by comparison with the weights of standard pieces of known area. In each section the number of cartilage-bearing bronchi present, together with the number and size of the fragments of cartilage related to each bronchus, were noted. The results from the Quantimet and the cutting out and weighing method were compared in nine cases (Table). It was found that the Quantimet gave lower readings than the projection micro-

T A B L E

\begin{tabular}{c|c|c}
\hline \multirow{2}{*}{$\begin{array}{c}\text { Age at Death } \\
\text { (weeks) }\end{array}$} & \multicolumn{2}{|c}{ Area of Cartilage $\left(\mathrm{mm}^{2}\right)$} \\
\hline & Quantimet & Projection Microscope \\
\hline 35 & 0.001 & 0.004 \\
38 & 0.36 & 0.67 \\
40 & 0.25 & 0.30 \\
40 & 0.13 & 0.16 \\
40 & 0.31 & 0.39 \\
40 & 0.24 & 0.26 \\
52 & 0.46 & 0.82 \\
56 & 0.28 & 0.38 \\
$412\left(6 \frac{1}{2} \mathrm{yr}\right)$ & 0.09 & 0.19 \\
\hline
\end{tabular}

scope because the variability in staining properties of the very young cartilage caused small fragments of cartilage to be visually identified but not isolated densitometrically. It would seem that the Quantimet was identifying a chemical quality of the cartilage rather than its presence or absence. It was therefore felt that the cutting out and weighing method was more convenient and accurate, and all the data presented in the charts and diagrams in this paper were obtained in this way.

The age and cause of death of the children were not known at the time of the quantitative lung study but these were later correlated with the clinical histories of the children. The gestational period was included in the age rating of the child, that is, a child born at term and dying three weeks later was 43 weeks of age. An immature stillborn was rated as its gestational age, this being the obstetrical estimate confirmed by the histological assessment of the other tissues in the body, such as the kidney and brain.

\section{RESULTS}

The range of values at different ages showed a variability which is seen in all developmental studies of children's tissues.

No attempt was made to exclude any of the lung sections measured on a pathological basis as exclusion in this series of material seemed to be unjustifiable. There is a virtual absence of completely normal tissues available for study in children of this age group. The number of normal children dying immediately from an accident is too small to give a reliable indication of the variability expected at any age, and this applies in almost equal measure to stillborn tissues. Also our studies of the lungs of children found unexpectedly dead, which in the past we would have considered normal, suggest that there are increasing numbers of aberrations which are now identifiable from normality, as shown by the lymphatic tissue (Emery and Dinsdale, 1972) and the blood vessels (Naeye, 1973).

A summary of the findings is shown in Fig. 1 where the mean lines are related to the gestational ages of the children. The percentiles of total cartilage related to crown-rump length are presented in Figure 2.

TOTAL CARTILAGE The amount of cartilage shows a progressive increase throughout childhood, the

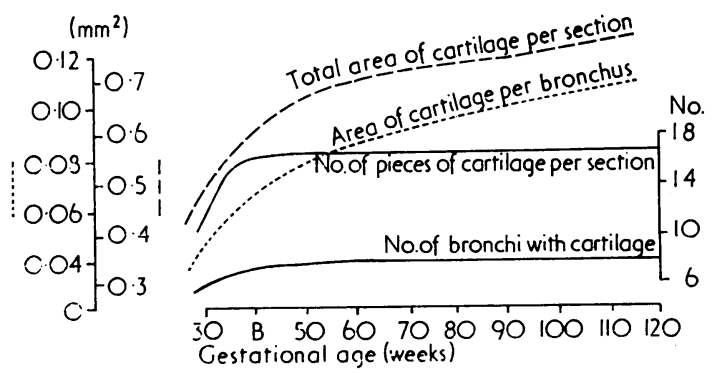

FIG. 1. Mean lines of cartilage growth related to gestational age. The total area of cartilage per bronchus and per section increases progressively with age, but the number of bronchi developing cartilage increases only until about 2 months of age. 


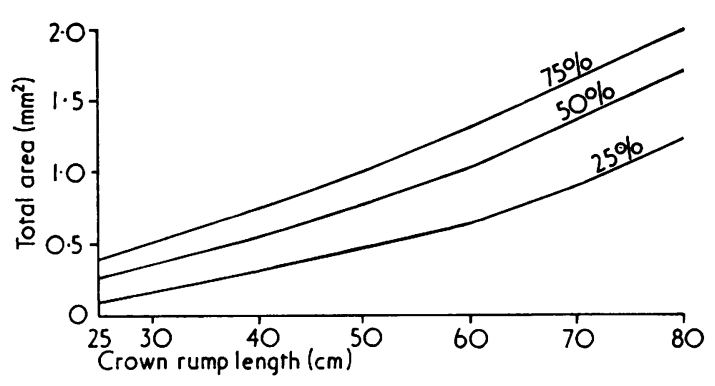

FIG. 2. When the total area of cartilage is related to crown-rump length, an almost straight line correlation is obtained.

increase being most rapid before the gestational age of $\mathbf{4 5}$ weeks, diminishing progressively for the next two months. Thereafter a fairly steady rate of growth occurs. When this total growth of cartilage is related to crown-rump length (Fig. 2) there appears to be an almost straight line correlation, which suggests that the proliferation of cartilage in the lungs parallels total body growth or, more specifically, the growth of the spine.

NUMBER OF CARTILAGE FRAGMENTS The total number of pieces of cartilage in a complete cross section of the lung (Fig. 1) shows a steep rise in children up to the gestational age of 40 weeks, but after 48 weeks there is virtually no increase in the total number of fragments seen.

CARTILAGE-BEARING BRONCHI The total number of air tubes bearing cartilage shows a progressive increase until the age of approximately 2 months postnatal (48 weeks gestational age) after which time the number of such bronchi appears to remain constant.

AVERAge area OF CARTILAGE PER BRONCHUS The average area of cartilage per bronchus shows a growth curve which is essentially parallel to the total amount of cartilage in the section.

\section{DISCUSSION}

The development curves that we have obtained from the right middle lobes of the lung differ in some aspects from the results obtained by Bucher and Reid (1961). Our findings suggest that there is a continuous production of fresh pieces of cartilage in the bronchi until the age of 48 weeks, that is, two months beyond normal term. This is shown by the increasing numbers of both cartilage-bearing bronchi and fragments of cartilage seen in the sections. After two months no further increase was seen.
The apparent conflict between the statemento by Bucher and Reid (1961) that no more cartilage을 develops after the 25th intrauterine week and ours? findings is not surprising, as their studies were carried out in a completely different way andw using a quite different population. Bucher and Reid did a detailed study of 14 fetuses between the ages of 10 weeks and term and they appeared $\vec{\omega}$ to have been uncertain about the gestational age of two of their stated term babies. Their studies, were not designed to indicate the range of growth likely to be found in a larger population but simply to indicate a general pattern of develop ment. Our findings confirm their general concepb of the production of almost all cartilages before birth but suggest that there is no clear cut-oft point for this and that development continues until term and for several weeks after birth.

The average area of cartilage shows a growt10 curve which is essentially parallel to the sitting height of the child; the variability of our data about the mean was considerably less when the total amount of cartilage was related to the crown-rump length than when it was related tos gestational age. This suggests that the growth of pulmonary cartilage can be influenced by the same factors that govern the growth of genera $B$ body cartilage, and thus the use of this criterion $\overrightarrow{5}$ will express only relative pulmonary cartilage deficiency. The 25th, 50th, and 75th percentile lines are presented as working diagrams ing. Figure 2.

Our data are derived from a complete cross section of apparently anatomically normal lungs from children dying from a wide variety o $\hat{\underline{Q}}$ causes, thus our percentiles represent the usual and not the normal. We do not know what degree of relative overgrowth or undergrowth of cartil age affects the stability of the lung, or is related to liability to respiratory symptoms or infection $>$ As there seems to be a direct relationship be을. tween the length of the child and the growth of the bronchial cartilage, it seems likely that factors related to decreased growth in height coulds similarly affect the lung. Our figures show a widet variability in those below than those above the 50th percentile levels, and this suggests that the lower group contains lungs in which there may have been pathological delay in cartilage growth ${ }^{\text {? }}$ Thus it seems worth while to study such lungș from the point of view of detecting minor degree? of bronchomalacia.

This work was partly supported by the Foundation for the Study of Infant Deaths, by ASBAH, The 
South African Medical Research Council, and the Richard Fund.

\section{REFERENCES}

Bucher, U. (1969). Development of cartilage. In The Anatomy of the Developing Lung, by J. L. Emery. William Heinemann Medical Books, London.

- and Reid, L. (1961). Development of the intrasegmental bronchial tree: The pattern of branching and development of cartilage at various stages of intra-uterine life. Thorax, 16, 729.

Emery, J. L. and Dinsdale, F. (1972). The postnatal development of lympho-reticular aggregates and lymph nodes in infants' lungs. Journal of Clinical Pathology, 26, 539. $\longrightarrow$ and Wilcock, P. F. (1966). The postnatal development of the lung. Acta Anatomica, 65, 10.

von Hayek, H. (1960). The Human Lung. Hafner Publishing Company, New York.

Landing, B. H. and Wells, T. H. (1973). Tracheobronchial abnormalities in children. In Perspectives of Pediatric Pathology, Volume I, edited by Harvey, S. Rosenberg and Robert P. Bolande. Year Book Medical Publishers, Chicago.

Naeye, R. L. (1973). Pulmonary arterial abnormalities in the sudden-infant-death-syndrome. New England Journal of Medicine, 289, 1187.

Requests for reprints to: Dr. C. C. Sinclair-Smith, Red Cross War Memorial Children's Hospital, Rondebosch, Cape Town, South Africa (7700). 